\title{
THE LIFTING OF THE UKK PROPERTY FROM $E$ TO $C_{E}$
}

\author{
YU-PING HSU
}

(Communicated by Dale Alspach)

\begin{abstract}
In this paper we show that $C_{E}$, the unitary matrix space associated with the symmetrically normed sequence space $E$, has the $U K K$ property for the weak operator topology if $E$ has the $U K K$ property for the pointwise convergence topology. We also prove that the quasi-normed space $C_{p}=C_{l_{p}}$, for $0<p<1$, has the $U K K$ property for the weak operator topology.
\end{abstract}

\section{INTRODUCTION}

Let $\Phi$ be a symmetric norm on $F$, the space of infinite sequences with only finitely many nonzero elements, i.e., $\Phi$ is invariant under permutations and depends only on the absolute values of coordinates. The maximal symmetric sequence space associated to $\Phi$, denoted by $E_{\Phi}$, is defined by

$$
E_{\Phi}=\left\{x: \lim _{n \rightarrow \infty} \Phi\left(x_{1}, x_{2}, \ldots, x_{n}, 0,0, \ldots\right)<\infty\right\}
$$

with norm $\|x\|_{E_{\Phi}}=\lim _{n \rightarrow \infty} \Phi\left(x_{1}, x_{2}, \ldots, x_{n}, 0,0, \ldots\right)$. The minimal symmetric sequence space associated to $\Phi$, denoted by $E_{\Phi}^{(0)}$, is defined to be the closure (in $E_{\Phi}$ ) of $F$.

Let $E$ be a general symmetric sequence space lying between $E_{\Phi}^{(0)}$ and $E_{\Phi}$. The unitary matrix space $C_{E}$ associated with $E$ is the Banach space of all compact operators on $l_{2}$ for which $\lambda(A) \in E$, normed by $\Phi(A)=\|(\lambda(A))\|_{E}$. Here $\lambda(A)=\left(\lambda_{n}(A)\right)$ is the sequence of $s$-numbers of $\mathrm{A}$, i.e., the eigenvalues of $\left(A^{*} A\right)^{1 / 2}$ arranged in a nonincreasing ordering counting multiplicity.

Let $(X,\|\cdot\|)$ be a Banach space, and let $\tau$ be a topological vector space topology on $X$ that is weaker than the norm topology.

Definition 1.1. $X$ is said to have the Kadec-Klee property with respect to $\tau$, denoted by $K K(\tau)$, if whenever $\left\{x_{n}\right\}_{n=1}^{\infty}$ is a sequence in $X$ such that $x_{n} \rightarrow$ $x \in X$ with respect to $\tau$ and $\left\|x_{n}\right\| \rightarrow\|x\|$, then it follows that $\left\|x_{n}-x\right\| \rightarrow 0$.

Definition 1.2. $X$ is said to have the uniform Kadec-Klee property with respect to $\tau$, denoted by $U K K(\tau)$, if for every $\varepsilon>0$ there exists $\delta(\varepsilon)>0$ so that

Received by the editors April 28, 1993 and, in revised form, November 5, 1993.

1991 Mathematics Subject Classification. Primary 47D25, 46B20; Secondary 47H10.

Key words and phrases. Kadec-Klee, fixed point property, unitary matrix space, symmetric sequence space. 
whenever $\left\{x_{n}\right\}_{n=1}^{\infty}$ is a sequence in the unit ball of $X$ such that $x_{n} \rightarrow x \in X$ with respect to $\tau$ and $\inf _{n \neq m}\left\|x_{n}-x_{m}\right\| \geq \varepsilon$, then it follows that $\|x\| \leq 1-\delta(\varepsilon)$.

It is easy to show that the $U K K$ property implies the $K K$ property.

For a symmetric sequence space $E$ and its associated unitary matrix space $C_{E}$ we are interested in the topology of pointwise convergence and the weak operator topology respectively.

Arazy [1] and Simon [14] show that if $E$ has the $K K$ property with respect to the pointwise convergence topology, then $C_{E}$ has the $K K$ property with respect to the weak operator topology. Our main result (Theorem 3.1) is the analogous result for the $U K K$ property. As a consequence of the main result, $C_{E}$ has a fixed point property for nonexpansive mappings if $E$ has the $U K K$ property. We must also mention here the recent interesting results in [2] concerning relations between the $U K K$ property for a symmetric Banach function space $E$ and the corresponding space $E(\mathscr{M})$ of all $\tau$-measurable operators affiliated with a von Neumann algebra $\mathscr{M}$ that supports a faithful, normal, semi-finite trace $\tau$ whose decreasing rearrangement lies in $E$ : if $E$ is $\alpha$-convex with constant 1 for some $0<\alpha \leq 1$ and if $E$ satisfies a lower- $q$ estimate with constant 1 for some finite $q \geq \alpha$, then $E(\mathscr{M})$ has the $U K K$ property for the topology of local convergence in measure. As a special case of this result it follows that $C_{p}(0<p<1)$ has the $U K K$ property for the weak operator topology. In Section 4 below we present a short elementary proof of this fact.

This paper is part of my Ph.D. dissertation. I thank my adviser Stephen Dilworth for his guidance. And I also thank Chris Lennard for sending me the preprint [2] and for his helpful comments.

\section{Preliminaries}

The following definition is useful in studying the $U K K$ property.

Definition 2.1. The $U K K(\tau)$-modulus of a space $X$ is defined by $\delta_{X}(\varepsilon)=$ $\inf \{1-\|x\|\}$, where the infimum is taken over all $x$ such that $x$ is the $\tau$-limit of a sequence $\left\{y_{n}\right\}$ in the unit ball of $X$ with $\inf _{n \neq m}\left\|y_{n}-y_{m}\right\| \geq \varepsilon$.

Lemma 2.2. If $E$ has the $K K$ property for the topology of pointwise convergence then $E$ is minimal (i.e., $E=E_{\Phi}^{(0)}$ ).

Proof. Let $x \in E$. Then $x^{(n)}=\left(x_{1}, x_{2}, \ldots, x_{n}, 0,0, \ldots\right) \rightarrow x$ pointwise and $\left\|x^{(n)}\right\|_{E} \rightarrow\|x\|_{E}$. By the $K K$ property, $\left\|x^{(n)}-x\right\|_{E} \rightarrow 0$; hence $x \in E_{\Phi}^{(0)}$.

Lemma 2.3. If $E$ has the $U K K$ property for the topology of pointwise convergence then $E$ is maximal (i.e., $E=E_{\Phi}$ ).

Proof. Suppose that $E$ is not maximal. Then $\left(e_{n}\right)_{n=1}^{\infty}$ is not a boundedly complete Schauder basis (see [10, Definition 1.b.3]). Therefore by [10, Theorem 1.c.10] $E$ contains a subspace isomorphic to $c_{0}$. By [7, Lemma 2.2], for any $\varepsilon>0$, there is a sequence $\left\{y_{n}\right\}$ of elements of the unit ball such that

$$
(1-\varepsilon) \sup \left|a_{i}\right| \leq\left\|\sum a_{i} y_{i}\right\|_{E} \leq \sup \left|a_{i}\right|
$$

for all finite sequences $\left\{a_{n}\right\}$ of real numbers. Let $z_{n}=y_{1}+y_{n}$; then $\left\|z_{n}\right\|_{E} \leq 1$ and $\left\|z_{n}-z_{m}\right\|_{E} \geq 1-\varepsilon$. Clearly $y_{n} \rightarrow 0$ pointwise in $E$, and so $z_{n} \rightarrow y_{1}$ 
pointwise in $E$. Let $\delta(\cdot)$ denote the $U K K$-modulus for the topology of pointwise convergence. Then

$$
1-\delta(1-\varepsilon) \geq\left\|y_{1}\right\|_{E} \geq 1-\varepsilon,
$$

which is a contradiction for $\varepsilon>0$ sufficiently small.

Example 2.4. For every $x \in c_{0}$, define $\|x\|=\sum 2^{-n} x_{n}^{*}$ where $\left(x_{n}^{*}\right)$ is the decreasing rearrangement of $\left(\left|x_{n}\right|\right)$. Then $\left(c_{0},\|\cdot\|\right)$ has the $K K$ property but not the $U K K$ property for the topology of pointwise convergence. Since $c_{0}$ is not maximal, it follows that Lemma 2.3 breaks down if " $U K K$ " is replaced by " $K K$ ".

Proof. Let $\left\{x^{(k)}\right\}$ be a sequence in $c_{0}$ converging pointwise to $x \in c_{0}$ and with $\left\|x^{(k)}\right\| \rightarrow\|x\|$. Without loss of generality we may assume that $x=\left(x_{n}^{*}\right)$ and that $\left\|x^{(k)}\right\|=\|x\|=1$. Given $\varepsilon>0$, there exists $N$ such that $x_{i}<\varepsilon / 2$ for all $i>N$, and so $\left\|\left(x_{1}, x_{2}, \ldots, x_{N}, 0,0, \ldots\right)\right\| \geq 1-2^{-(N+1)} \varepsilon$. Since $x^{(k)} \rightarrow x$ pointwise, there exists $M$ such that

$$
\left\|\left(x_{1}^{(k)}, x_{2}^{(k)}, \ldots, x_{N}^{(k)}, 0,0, \ldots\right)-\left(x_{1}, x_{2}, \ldots, x_{N}, 0,0, \ldots\right)\right\|<2^{-(N+1)} \varepsilon
$$

for all $k>M$. Thus,

$$
\left\|\left(x_{1}^{(k)}, x_{2}^{(k)}, \ldots, x_{N}^{(k)}, 0,0, \ldots\right)\right\|>1-2^{-N} \varepsilon
$$

for all $k>M$. By an obvious rearrangement inequality, for all $k>M$ we have

$$
\begin{aligned}
& \left\|\left(x_{1}^{(k)}, x_{2}^{(k)}, \ldots, x_{N}^{(k)}, 0,0, \ldots\right)\right\|+2^{-N}\left\|\left(x_{N+1}^{(k)}, x_{N+2}^{(k)}, \ldots, x_{n}^{(k)} \ldots\right)\right\| \\
& \quad \leq\left\|x^{(k)}\right\| \leq 1 .
\end{aligned}
$$

Hence $\left\|\left(x_{N+1}^{(k)}, x_{N+2}^{(k)}, \ldots, x_{n}^{(k)}, \ldots\right)\right\| \leq \varepsilon$ for all $k>M$. Thus,

$$
\begin{aligned}
\left\|x^{(k)}-x\right\| \leq & \left\|\left(x_{1}^{(k)}, x_{2}^{(k)}, \ldots, x_{N}^{(k)}, 0,0, \ldots\right)-\left(x_{1}, x_{2}, \ldots, x_{N}, 0,0, \ldots\right)\right\| \\
& +\left\|\left(x_{N+1}^{(k)}, x_{N+2}^{(k)}, \ldots, x_{n}^{(k)}, \ldots\right)\right\|+\left\|\left(x_{N+1}, x_{N+2}, \ldots, x_{n}, \ldots\right)\right\| \\
\leq & \left(2^{-(N+1)}+1+2^{-1}\right) \varepsilon<2 \varepsilon
\end{aligned}
$$

for all $k>M$. So $x^{(k)} \rightarrow x$ in norm. Therefore, $c_{0}$ has the $K K$ property with respect to the topology of pointwise convergence. It is interesting to note that by $[1,14]$ this symmetric $K K$ norm on $c_{0}$ will lift to a symmetric $K K$ norm on the ideal of compact operators on $l_{2}$.

In the following $\delta_{E}$ denotes the $U K K$-modulus for the topology of pointwise convergence in a symmetric sequence space $E$. We estimate $\delta_{E}$ in terms of a geometrical quantity $\beta_{E}$.

Proposition 2.5. Let $E$ be a symmetric sequence space with the $K K$ property, and let

$\beta_{E}(\varepsilon)=\inf \left\{1-\|x\|_{E}:\|x+y\|_{E} \leq 1, x, y\right.$ disjoint and $\left.\|y\|_{E} \geq \varepsilon, x, y \in E\right\}$.

Then $\delta_{E}(\varepsilon / 2) \leq \beta_{E}(\varepsilon) \leq \delta_{E}(2 \varepsilon)$.

Proof. Given $\eta>0$, choose $x, y \in E$ disjoint with $\|x+y\|_{E} \leq 1,\|y\|_{E} \geq \varepsilon$ such that $1-\|x\|_{E}<\beta_{E}(\varepsilon)+\eta$. Since $E$ is minimal, we can choose $N$ such that

$$
\left\|\left(x_{1}, x_{2}, \ldots, x_{N}, 0,0, \ldots\right)-x\right\|_{E}<\eta
$$


and

Define for $k=1,2,3, \ldots$,

$$
\left\|\left(y_{1}, y_{2}, \ldots, y_{N}, 0,0, \ldots\right)\right\|_{E}>\varepsilon / 2 .
$$

$$
z^{(k)}=(x_{1}, x_{2}, \ldots, x_{N}, \underbrace{0,0, \ldots, 0}_{k N \text { times }}, y_{1}, y_{2}, \ldots, y_{N}, 0,0, \ldots) .
$$

Then

$$
\left\|z^{(k)}\right\|_{E} \leq\|x+y\|_{E} \leq 1, \quad\left\|z^{(k)}-z^{(j)}\right\|_{E}>\varepsilon / 2, \quad j \neq k,
$$

and $z^{(k)} \rightarrow\left(x_{1}, x_{2}, \ldots, x_{N}, 0,0, \ldots\right)$ in the pointwise convergence topology. Therefore by the definition of the modulus we have

$$
\begin{aligned}
\delta_{E}(\varepsilon / 2) & \leq 1-\left\|\left(x_{1}, x_{2} \ldots, x_{N}, 0,0, \ldots\right)\right\|_{E} \\
& \leq 1-\|x\|_{E}+\eta \\
& \leq \beta_{E}(\varepsilon)+2 \eta .
\end{aligned}
$$

Since $\eta$ is arbitrary, $\delta_{E}(\varepsilon / 2) \leq \beta_{E}(\varepsilon)$. Given $\eta>0$, choose $x,\left\{y^{(n)}\right\}$ with $\left\|y^{(n)}\right\|_{E} \leq 1, \inf _{m \neq n}\left\|y^{(n)}-y^{(m)}\right\|_{E} \geq 2 \varepsilon$, and $y^{(n)} \rightarrow x$ pointwise, such that $1-\|x\|_{E} \leq \delta_{E}(2 \varepsilon)+\eta$. Since $y^{(n)} \rightarrow x$ pointwise, there exists $M>0$ such that for all $m>M$, we have (where $N$ is chosen as before)

$$
\left\|\left(y_{1}^{(m)}, y_{2}^{(m)}, \ldots, y_{N}^{(m)}, 0,0, \ldots\right)-\left(x_{1}, x_{2}, \ldots, x_{N}, 0,0, \ldots\right)\right\|_{E}<\eta .
$$

Therefore, for all $m>M$,

$$
\left\|\left(y_{1}^{(m)}, y_{2}^{(m)}, \ldots, y_{N}^{(m)}, 0,0, \ldots\right)-x\right\|_{E}<2 \eta .
$$

Since $\inf _{n \neq m}\left\|y^{(m)}-y^{(n)}\right\|_{E} \geq 2 \varepsilon$, there are infinitely many $y^{(m)}$ such that

$$
\left\|y^{(m)}-x\right\|_{E} \geq \varepsilon
$$

Hence $\left\|y^{(m)}-\left(y_{1}^{(m)}, \ldots, y_{N}^{(m)}, 0,0, \ldots\right)\right\|_{E} \geq \varepsilon-2 \eta$ for such $m$. Therefore

$$
\begin{aligned}
\beta_{E}(\varepsilon-2 \eta) & \leq 1-\left\|\left(y_{1}^{(m)}, y_{2}^{(m)}, \ldots, y_{N}^{(m)}, 0,0, \ldots\right)\right\| \\
& \leq 1-\|x\|_{E}+2 \eta \\
& \leq \delta_{E}(2 \epsilon)+3 \eta .
\end{aligned}
$$

Since $\eta$ is arbitrary, we have $\beta_{E}(\varepsilon) \leq \delta_{E}(2 \varepsilon)$.

The proof of the following lemma may be found in Simon [14].

Lemma 2.6. Let $\Phi$ be an arbitrary symmetric norm.

(a) If $P$ is an orthogonal projection and $Q=I-P$, and if $A \in C_{E}$, then $P A P+Q A Q \in C_{E}$ and $\Phi(P A P+Q A Q) \leq \Phi(A)$.

(b) If $A^{*} A$ and $B^{*} B$ lie in $C_{E}$, then $A^{*} B$ lies in $C_{E}$ and

$$
\Phi\left(A^{*} B\right) \leq \Phi\left(A^{*} A\right)^{1 / 2} \Phi\left(B^{*} B\right)^{1 / 2} .
$$

(c) If $A_{n}^{(j)} \rightarrow A^{(j)}, j=1, \ldots, k$, weakly with $A_{n}^{(j)}, A^{(j)} \in C_{E}$, then we can find an increasing sequence of finite rank projections $P_{n}$ with $\lim P_{n}=I$ in the strong operator topology such that $\Phi\left(P_{n} A_{n}^{(j)} P_{n}-A^{(j)}\right) \rightarrow 0, j=1, \ldots, k$.

Lemma 2.7. Let $A \in C_{E}$. If $P, Q$ are orthogonal projections, then

$$
\Phi(P A Q) \leq \Phi(|A|)^{1 / 2} \Phi(Q|A| Q)^{1 / 2} .
$$


Proof.

$$
\begin{aligned}
\Phi(P A Q) & \leq \Phi(A Q) \\
& =\Phi(W|A| Q)(\text { for some partial isometry } W) \\
& \leq \Phi(|A| Q)=\Phi\left(|A|^{1 / 2}|A|^{1 / 2} Q\right) \\
& \leq \Phi(|A|)^{1 / 2} \Phi(Q|A| Q)^{1 / 2}(\text { by lemma } 2.6(\mathrm{~b}))
\end{aligned}
$$

For a proof of the following lemma the reader is referred to [5, Theorem 5.1, Lemma 5.2].

Lemma 2.8. Let $\Phi$ be a symmetric norm, and let $E=E_{\Phi}$.

(a) $C_{E}$ coincides elementwise with $C_{\infty}$ the space of all compact operators if and only if $\lim _{n \rightarrow \infty} \Phi(\underbrace{1,1, \ldots, 1}_{n}, 0,0, \ldots)<\infty$.

(b) Suppose $C_{E}$ does not coincide elementwise with $C_{\infty}$. Whenever a bounded operator $A$ is the weak limit of a sequence of operators $\left\{A_{m}\right\}_{1}^{\infty}$ from $C_{E}$ such that $\sup _{m} \Phi\left(A_{m}\right)<\infty$, then $A$ also belongs to $C_{E}$, and $\Phi(A) \leq \sup _{m} \Phi\left(A_{m}\right)$.

Proposition 2.9. If a symmetric space $(E, \Phi)$ has the $U K K$ property for the topology of pointwise convergence, then the closed unit ball of $C_{E}$ is sequentially compact for the weak operator topology.

Proof. Let $\left\langle A_{n}\right\rangle$ be a sequence in the closed unit ball of $C_{E}$ and let $\left\{\varphi_{i}\right\}$ be an orthonormal basis for the underlying Hilbert space $H$. Then for fixed $i, j$ each sequence $\left\langle\left(A_{n} \varphi_{i}, \varphi_{j}\right)\right\rangle_{n=1}^{\infty}$ lies in the closed inteval $[-1,1]$, since

$$
\begin{aligned}
\left|\left(A_{n} \varphi_{i}, \varphi_{j}\right)\right| & \leq\left\|A_{n} \varphi_{i}\right\| \cdot\left\|\varphi_{j}\right\| \leq\left\|A_{n}\right\| \cdot\left\|\varphi_{i}\right\| \cdot\left\|\varphi_{j}\right\| \\
& \leq\left\|A_{n}\right\| \leq \Phi\left(A_{n}\right) \leq 1 .
\end{aligned}
$$

By a diagonal process there is a subsequence $\left\langle A_{n_{k}}\right\rangle$ and a bounded operator $A$ such that $\left(A_{n_{k}} \varphi_{i}, \varphi_{j}\right) \rightarrow\left(A \varphi_{i}, \varphi_{j}\right)$ for all $i, j$; in particular, $A_{n_{k}} \rightarrow$ $A$ in the weak operator topology. Suppose, to derive a contradiction, that $\sup _{n} \Phi(\underbrace{1,1, \ldots, 1}_{n}, 0,0, \ldots)=\infty$. Since $\Phi$ has the $U K K$ property, $E$ is maximal by Lemma 2.3. Thus $x=(1,1, \ldots, 1, \ldots) \in E$, and so $x_{k}=$ $(\underbrace{1,1, \ldots, 1}_{k}, 0,0, \ldots)$ converges to $x$ pointwise and $\Phi\left(x_{k}\right) \rightarrow \Phi(x)$, which implies that $\Phi\left(x_{k}-x\right) \rightarrow 0$ by the $K K$ property for $E$; but $\Phi\left(x_{k}-x\right)=$ $\Phi(\underbrace{0,0, \ldots, 0}_{k}, 1,1, \ldots) \geq 1$ for all $\mathrm{k}$, which is the desired contradiction.

Hence, by Lemma 2.8(a), $C_{E}$ and $C_{\infty}$ do not coincide elementwise, and by Lemma 2.8(b), $A$ is in the closed unit ball of $C_{E}$.

Remark 2.10. Example 2.4 shows that Proposition 2.9 breaks down if " $U K K$ " is replaced by " $K K$ ".

\section{3. $U K K$ PROPERTY FOR $E$ IMPLIES $U K K$ PROPERTY FOR $C_{E}$}

Theorem 3.1. If a symmetric sequence space $E$ with norm $\Phi$ has the $U K K$ property for the pointwise convergence topology, then $C_{E}$ has the UKK property 
for the weak operator topology. Moreover, if $\delta_{E}$ and $\delta_{C_{E}}$ denote the corresponding $U K K$-moduli for $E$ and $C_{E}$ respectively, then $\delta_{C_{E}}(\varepsilon) \geq \frac{1}{2} \delta_{E}\left(\varepsilon^{2} / 128\right)$.

Proof. Suppose $\left\langle A_{n}\right\rangle$ is a sequence in the the unit ball of $C_{E}$ and that $A_{n} \rightarrow A$ in the weak operator topology and $\inf _{n \neq m} \Phi\left(A_{m}-A_{n}\right) \geq \varepsilon>0$. Without loss of generality we may assume $\left|A_{n}\right| \stackrel{w}{\longrightarrow} B,\left|A_{n}^{*}\right| \stackrel{w}{\longrightarrow} C$ for some $B$ and $C$ belong to the closed unit ball of $C_{E}$, since by Proposition 2.9 the unit ball of $C_{E}$ is sequentially compact. By Lemma 2.6(c) there is a sequence $P_{n}$ of finite rank orthogonal projections such that $P_{n} \uparrow I$ strongly, $\Phi\left(P_{n} A_{n} P_{n}-A\right) \rightarrow 0$, $\Phi\left(P_{n}\left|A_{n}\right| P_{n}-B\right) \rightarrow 0$, and $\Phi\left(P_{n}\left|A_{n}^{*}\right| P_{n}-C\right) \rightarrow 0$. Let $Q_{n}=I-P_{n}$. Since there are infinitely many $A_{n}$ with $\Phi\left(A_{n}-A\right) \geq \varepsilon / 2$, we may assume, by passing to a subsequence, that

$$
\begin{aligned}
\frac{\varepsilon}{2} \leq \Phi\left(A_{n}-A\right) \leq & \Phi\left(P_{n} A_{n} P_{n}-A\right)+\Phi\left(P_{n} A_{n} Q_{n}\right) \\
& +\Phi\left(Q_{n} A_{n} Q_{n}\right)+\Phi\left(Q_{n} A_{n} P_{n}\right)
\end{aligned}
$$

for all $n$. Since $\Phi\left(P_{n} A_{n} P_{n}-A\right) \rightarrow 0$, one of the following must hold:

(i) $\Phi\left(Q_{n} A_{n} Q_{n}\right) \geq \varepsilon / 8$ for infinitely many $n$;

(ii) $\Phi\left(P_{n} A_{n} Q_{n}\right) \geq \varepsilon / 8$ for infinitely many $n$;

(iii) $\Phi\left(Q_{n} A_{n} P_{n}\right) \geq \varepsilon / 8$ for infinitely many $n$.

By passing to a subsequence, we may suppose that one of the three cases holds for all $n$.

Case 1. Suppose (i) holds for all $n$. Define $x^{(1)}$ to be the sequence obtained by first listing all the singular numbers of $P_{1} A_{1} P_{1}$, including enough zeros to have $\operatorname{dim} P_{1}$ entries, and then after that listing finitely many singular numbers of $Q_{1} A_{1} Q_{1}$ so that $\Phi\left(\lambda_{1}\left(Q_{1} A_{1} Q_{1}\right), \ldots, \lambda_{s_{1}}\left(Q_{1} A_{1} Q_{1}\right)\right) \geq \varepsilon / 16$, and finally zeros after that.

Now suppose that $x^{(j-1)}$ has been defined. We define $x^{j}$ inductively by first listing all the singular numbers of $P_{n_{j}} A_{n_{j}} P_{n_{j}}$, where $n_{j}>n_{j-1}$ is chosen such that $n_{j}$ is the least number such that $\operatorname{dim} P_{n_{j}}>$ the length of $x^{(j-1)}$ (here the length of $x^{(j)}$ is defined to equal $\left.\min \left\{n: x_{i}^{(j)}=0 \forall i>n\right\}\right)$, and then listing finitely many singular number of $Q_{n_{j}} A_{n_{j}} Q_{n_{j}}$ such that

$$
\Phi\left(\lambda_{1}\left(Q_{n_{j}} A_{n_{j}} Q_{n_{j}}\right), \lambda_{2}\left(Q_{n_{j}} A_{n_{j}} Q_{n_{j}}\right), \ldots, \lambda_{s_{j}}\left(Q_{n_{j}} A_{n_{j}} Q_{n_{j}}\right)\right) \geq \varepsilon / 16
$$

By Lemma 2.6(a), we have

$$
\Phi\left(x^{(j)}\right) \leq \Phi\left(P_{n_{j}} A_{n_{j}} P_{n_{j}}+Q_{n_{j}} A_{n_{j}} Q_{n_{j}}\right) \leq \Phi\left(A_{n_{j}}\right) \leq 1 .
$$

Thus we have defined a sequence $\left\langle x^{(n)}\right\rangle$ in the unit ball of $E$. Let $x$ be the sequence of singular numbers of $A$. Now $\left\|P_{n} A_{n} P_{n}-A\right\| \rightarrow 0$, and so $x_{i}^{(j)} \rightarrow x_{i}$; also, for $j>i$,

$$
\Phi\left(x^{(i)}-x^{(j)}\right) \geq \varepsilon / 16
$$

Since $E$ has the $U K K$ property, we have $\Phi(x) \leq 1-\delta_{E}(\varepsilon / 16)$. So $\Phi(A)=$ $\Phi(x) \leq 1-\delta_{E}(\varepsilon / 16)$.

Case 2. Suppose (ii) holds for all $n$. By Lemma 2.7

$$
\Phi\left(P_{n} A_{n} Q_{n}\right) \leq \boldsymbol{\Phi}\left(\left|A_{n}\right|\right)^{1 / 2} \Phi\left(Q_{n}\left|A_{n}\right| Q_{n}\right)^{1 / 2},
$$

i.e. $\Phi\left(Q_{n}\left|A_{n}\right| Q_{n}\right) \geq \Phi\left(P_{n} A_{n} Q_{n}\right)^{2} \geq \varepsilon^{2} / 64$. 
Define $x^{(j)}$ in the same way as in Case 1 , changing $A_{n_{j}}$ to $\left|A_{n_{j}}\right|, A$ to $B$, and $\varepsilon / 8$ to $\varepsilon^{2} / 64$. We obtain $\Phi(B)=\Phi(x) \leq 1-\delta_{E}\left(\varepsilon^{2} / 128\right)$. But by Lemma 2.7, $\Phi\left(P_{n} A_{n} P_{n}\right)^{2} \leq \Phi\left(P_{n}\left|A_{n}\right| P_{n}\right)$, and we have

$$
\Phi(A)=\lim _{j \rightarrow \infty} \Phi\left(P_{n_{j}} A_{n_{j}} P_{n_{j}}\right) \leq \lim _{j \rightarrow \infty} \Phi\left(P_{n_{j}}\left|A_{n_{j}}\right| P_{n_{j}}\right)^{1 / 2}=\Phi(B)^{1 / 2} .
$$

So $\Phi(A) \leq \Phi(B)^{1 / 2} \leq \sqrt{1-\delta_{E}\left(\varepsilon^{2} / 128\right)} \leq 1-\frac{1}{2} \delta_{E}\left(\varepsilon^{2} / 128\right)$.

Case 3. Suppose (iii) holds for all $n$. Since $\Phi\left(Q_{n} A_{n} P_{n}\right)=\Phi\left(P_{n} A_{n}^{*} Q_{n}\right)$, Lemma 2.7 again gives

$$
\Phi\left(Q_{n}\left|A_{n}^{*}\right| Q_{n}\right) \geq \Phi\left(Q_{n} A_{n} P_{n}\right)^{2} \geq \varepsilon^{2} / 64
$$

Similarly we will get

$$
\begin{aligned}
\Phi(A) & =\lim _{j \rightarrow \infty} \Phi\left(P_{n_{j}} A_{n_{j}} P_{n_{j}}\right) \\
& \leq \lim _{j \rightarrow \infty} \Phi\left(P_{n_{j}}\left|A_{n_{j}}^{*}\right| P_{n_{j}}\right)^{1 / 2} \\
& =\Phi(C) \leq 1-\frac{1}{2} \delta_{E}\left(\varepsilon^{2} / 128\right) .
\end{aligned}
$$

Hence $C_{E}$ has the $U K K$ property for the weak operator topology and $\delta_{C_{E}}(\varepsilon) \geq$ $\frac{1}{2} \delta_{E}\left(\varepsilon^{2} / 128\right)$.

Remark. It is clear that $\beta_{l_{1}}=\varepsilon$. By Proposition 2.5, $\delta_{l_{1}}(\varepsilon) \geq \varepsilon / 2$ (in fact $\left.\delta_{l_{1}}(\varepsilon)=\varepsilon / 2\right)$. So

$$
\delta_{C_{1}}(\varepsilon) \geq \frac{1}{2} \delta_{l_{1}}\left(\varepsilon^{2} / 128\right) \geq \frac{\varepsilon^{2}}{512}
$$

In Lennard [8] there is the better estimate $1-\delta_{C_{1}}(\varepsilon) \leq\left(1-(\varepsilon / 2 \sqrt{3})^{2}\right)^{1 / 2}$, which implies $\delta_{C_{1}}(\varepsilon) \geq \varepsilon^{2} / 24$.

Let $K$ be a closed bounded convex subset of a Banach space $(X,\|\cdot\|)$. A map $T: K \rightarrow K$ is said to be nonexpansive if $\|T x-T y\| \leq\|x-y\|$ for all $x, y \in K . K$ is said to have the fixed point property if it has a fixed point for every nonexpansive mapping. By van Dulst and Sims [3], we have the following corollary.

Corollary 3.2. If $E$ has the UKK property for the topology of pointwise convergence, then every convex subset of $C_{E}$ which is compact in the weak operator topology has the fixed point property.

Remark 3.3. It follows from Proposition 2.9 and the above corollary that if $E$ has the $U K K$ property, then the closed unit ball of $C_{E}$ has the fixed point property.

\section{THE $U K K$ PROPERTY IN $C_{p}$ FOR $0<p<1$}

First we give an example to show that Lemma 2.6(a) breaks down for quasinorms. Thus the proof of Theorem 3.1 appears to break down completely for quasi-norms.

Example 4.1. Let $p=\frac{1}{2}$,

$$
A=\left[\begin{array}{ll}
1 & 1 \\
1 & 1
\end{array}\right] \quad \text { and } \quad P=\left[\begin{array}{ll}
1 & 0 \\
0 & 0
\end{array}\right] \text {. }
$$


Then $A=|A|$ and $P$ is an orthogonal projection. Let $Q=I-P$, so that

$$
P A P+Q A Q=\left[\begin{array}{ll}
1 & 0 \\
0 & 1
\end{array}\right] .
$$

Hence $\|P A P+Q A Q\|_{p}=\left(1^{1 / 2}+1^{1 / 2}\right)^{2}=4$. But $\|A\|_{p}=\left(0^{1 / 2}+2^{1 / 2}\right)^{2}=2$. So we have $\|P A P+Q A Q\|_{p}>\|A\|_{p}$.

Proposition 4.2. Let $0<p \leq 2$, let $P$ be an orthogonal projection, and let $Q=I-P$. Then $\|A\|_{p}^{2} \geq\|P A\|_{p}^{2}+\|Q A\|_{p}^{2}$, and $\|A\|_{p}^{2} \geq\|A P\|_{p}^{2}+\|A Q\|_{p}^{2}$ for all $A \in C_{p}$.

Proof. By [6, Theorem 4], $\|A\|_{p}=\min \left(\sum\left\|A e_{n}\right\|^{p}\right)^{1 / p}$, where the minimum is taken over all orthonomal bases $\left(e_{n}\right)$. Let $\left(e_{n}\right)$ be an orthonormal basis such that $\|A\|_{p}=\left(\sum\left\|A e_{n}\right\|^{p}\right)^{1 / p}$. Let $f_{n}=P A e_{n}$ and $g_{n}=Q A e_{n}$; then

$$
\begin{aligned}
\|A\|_{p} & =\left(\sum\left\|A e_{n}\right\|^{p}\right)^{1 / p}=\left(\sum\left\|(P+Q) A e_{n}\right\|^{p}\right)^{1 / p} \\
& =\left(\sum\left\|P A e_{n}+Q A e_{n}\right\|^{p}\right)^{1 / p}=\left(\sum\left(\left\|f_{n}\right\|^{2}+\left\|g_{n}\right\|^{2}\right)^{p / 2}\right)^{1 / p} \\
& =\left[\left(\sum\left(\left\|f_{n}\right\|^{2}+\left\|g_{n}\right\|^{2}\right)^{p / 2}\right)^{2 / p}\right]^{1 / 2} \\
& \geq\left[\left\|\left\langle\left\|f_{n}\right\|^{2}\right\rangle\right\|_{p / 2}+\left\|\left\langle\left\|g_{n}\right\|^{2}\right\rangle\right\|_{p / 2}\right]^{1 / 2} \quad \text { (by the reverse Hölder inequality), } \\
& =\left[\left(\sum\left\|f_{n}\right\|^{p}\right)^{2 / p}+\left(\sum\left\|g_{n}\right\|^{p}\right)^{2 / p}\right]^{1 / 2} \\
& \geq\left[\|P A\|_{p}^{2}+\|Q A\|_{p}^{2}\right]^{1 / 2} .
\end{aligned}
$$

Consequently, $\|A\|_{p}^{2}=\left\|A^{*}\right\|_{p}^{2} \geq\left\|P A^{*}\right\|_{p}^{2}+\left\|Q A^{*}\right\|_{p}^{2}=\|A P\|_{p}^{2}+\|A Q\|_{p}^{2}$.

Remark. Applying Proposition 4.1 twice we obtain

$$
\|A\|_{p}^{2} \geq\|P A P\|_{p}^{2}+\|Q A P\|_{p}^{2}+\|P A Q\|_{p}^{2}+\|Q A Q\|_{p}^{2} .
$$

This inequality appears for the case $p=1$ in [1] and [8].

Theorem 4.3. The quasi-normed operator ideal $C_{p} \quad(0<p<1)$ has the UKK property with respect to the weak operator topology with $\delta_{C_{p}}(\varepsilon) \geq\left(2^{3-6 / p} / 3\right) \varepsilon^{2}$. Proof. Let $\left\langle A_{n}\right\rangle$ be a sequence in the unit ball of $C_{p}$ such that $\left\|A_{m}-A_{n}\right\|_{p} \geq \varepsilon$ $(m \neq n)$, and such that $A_{n} \rightarrow A$ in the weak operator topology. Let $\left\{P_{n}\right\}_{n=1}^{\infty}$ be finite rank orthogonal projections so that $P_{n} \uparrow I$ strongly and $\left\|P_{n} A_{n} P_{n}-A\right\|_{p} \rightarrow$ 0 .

Since $\left\|A_{n}-A_{m}\right\|_{p} \geq \varepsilon, m \neq n$, there is a subsequence $\left\langle A_{n_{k}}\right\rangle$ with

$$
\left\|A_{n_{k}}-A\right\|_{p} \geq \frac{\varepsilon}{2} \cdot 2^{1-\frac{1}{p}}=2^{\frac{-1}{p}} \cdot \varepsilon .
$$

Hence by the remark following Proposition 4.1,

$$
\begin{aligned}
2^{\frac{-1}{p}} \cdot \varepsilon & \leq\left\|A_{n_{k}}-A\right\|_{p} \\
& \leq 2^{\frac{2}{p}-2}\left\{\left\|P_{n_{k}} A_{n_{k}} P_{n_{k}}-A\right\|_{p}+\left\|P_{n_{k}} A_{n_{k}} Q_{n_{k}}\right\|_{p}\right. \\
& \left.\quad+\left\|Q_{n_{k}} A_{n_{k}} P_{n_{k}}\right\|_{p}+\left\|Q_{n_{k}} A_{n_{k}} Q_{n_{k}}\right\|_{p}\right\} \\
& \leq 2^{\frac{2}{p}-2}\left\{\left\|P_{n_{k}} A_{n_{k}} P_{n_{k}}-A\right\|_{p}+\sqrt{3}\left(\left\|A_{n_{k}}\right\|_{p}^{2}-\left\|P_{n_{k}} A_{n_{k}} P_{n_{k}}\right\|_{p}^{2}\right)^{1 / 2}\right\} .
\end{aligned}
$$

Taking the limit, we have

$$
2^{\frac{-1}{p}} \cdot \varepsilon \leq \sqrt{3} \cdot 2^{\frac{2}{p}-2}\left(1-\|A\|_{p}^{2}\right)^{1 / 2} .
$$


Thus

$$
2^{\frac{-2}{p}} \cdot \varepsilon^{2} \leq 3 \cdot 2^{\frac{4}{p}-4}\left(1-\|A\|_{p}^{2}\right)
$$

So

$$
\|A\|_{p} \leq\left(1-\frac{2^{4-\frac{6}{p}}}{3} \varepsilon^{2}\right)^{1 / 2} \leq 1-\frac{2^{3-\frac{6}{p}}}{3} \varepsilon^{2} .
$$

Therefore, $C_{p}$ has the $U K K$ property for the weak operator topology with

$$
\delta_{C_{p}}(\varepsilon) \geq \frac{2^{3-6 / p}}{3} \varepsilon^{2}
$$

\section{REFERENCES}

1. J. Arazy, More on convergence in unitary matrix spaces, Proc. Amer. Math. Soc. 83 (1981), 44-48.

2. P. G. Dodds, T. K. Dodds, P. N. Dowling, C. J. Lennard, and F. A. Sukochev, A uniform Kadec-Klee property for symmetric operator space, preprint, 1992.

3. D. van Dulst and B. Sims, Fixed points of non-expansive mappings and Chebyshev centers in Banach spaces with norms of type $(K K)$, Banach Space Theory and its Applications, Lecture Notes in Math., vol. 991, Springer-Verlag, New York, 1983, pp. 35-43.

4. N. Dunford and J. Schwartz, Linear operators, Vol. II. Spectral theory, Interscience, New York, 1963.

5. I. C. Gohberg and M. G. Krein, Introduction to the theory of linear nonselfadjoint operators, Transl. Math. Monographs, vol. 18, Amer. Math. Soc., Providence, RI, 1969.

6. I. C. Gohberg and A. S. Markus, Some relations between eigenvalues and matrix elements of linear operators, Mat. Sb. 64 (1964), 481-496; English transl., Amer. Math. Soc. Transl. 52 (1966), 201-216.

7. Robert C. James, Uniformly non-square Banach space, Ann. Math. 80 (1964), 542-550.

8. C. J. Lennard, $\mathscr{C}_{1}$ is uniformly Kadec-Klee, Proc. Amer. Math. Soc. 109 (1990), 71-77.

9. 100 (1991), 95-108.

10. J. Lindenstrauss and L. Tzafriri, Classical Banach space I, Springer-Verlag, Berlin, Heidelberg, and New York, 1977.

11. C. A. McCarthy, $C_{p}$, Israel J. Math. 5 (1967), 249-271.

12. R. Schatten, Norm ideals of completely continuous operators, Springer-Verlag, Berlin, 1960.

13. B. Simon, Trace ideals and their applications, Cambridge Univ. Press, Cambridge, 1979.

14. __ Convergence in trace ideals, Proc. Amer. Math. Soc. 83 (1981), 39-43.

Department of Mathematics, University of South Carolina, Columbia, South CarOLINA 29208

Current address: 66-2 Ln. 6 Tunghsin St., Keelung, Taiwan, Republic of China

E-mail address: B0219@ntou66.ntou.edu.tw 